\title{
Correction to: Treatment response scoring systems to assess long-term prognosis in self-injectable DMTs relapsing-remitting multiple sclerosis patients
}

\author{
Jordi Río ${ }^{1}$ - Àlex Rovira ${ }^{2}$. Claudio Gasperini ${ }^{3} \cdot$ Mar Tintoré $^{1} \cdot$ Luca Prosperini $^{3}$. Susana Otero-Romero ${ }^{1}$. \\ Manuel Comabella ${ }^{1}$ - Ángela Vidal-Jordana ${ }^{1}$ - Ingrid Galán ${ }^{1}$ - Luciana Midaglia ${ }^{1}$. Breogán Rodriguez-Acevedo ${ }^{1}$. \\ Ana Zabalza ${ }^{1}$. Joaquim Castilló ${ }^{1}$ - Georgina Arrambide ${ }^{1}$. Carlos Nos ${ }^{1}$ - Álvaro Cobo-Calvo ${ }^{1}$. Carmen Tur ${ }^{1}$. \\ Cristina Auger $^{2} \cdot$ Jaume Sastre-Garriga ${ }^{1} \cdot$ Xavier Montalban $^{1}$
}

Published online: 20 November 2021

(c) The Author(s), under exclusive licence to Springer-Verlag GmbH Germany 2021

\section{Correction to: Journal of Neurology}

https://doi.org/10.1007/s00415-021-10823-z

The original version of this article unfortunately contained a mistake. Author name Álvaro Cobo-Calvo was incorrectly written as Álvaro Cobo, therefore it is abbreviated wrongly as "A Cobo" in PubMed. The correct given name is Álvaro and family name is Cobo-Calvo.

The original article can be found online at https://doi.org/10.1007/ s00415-021-10823-z.

Jordi Río

jrio@cem-cat.org

1 Centre d'Esclerosi Múltiple de Catalunya (Cemcat), Servicio de Neurologia/Neuroimmunolgia, Hospital Universitari Vall d'Hebron, Passeig Vall d'Hebron 119-129, 08035 Barcelona, Spain

2 Sección de Neuroradiología, Servicio de Radiologia, Hospital Universitari Vall d'Hebron, Universitat Autònoma de Barcelona, Barcelona, Spain

3 Department of Neurosciences, San Camillo-Forlanini Hospital, Rome, Italy 